\title{
Marine Ecosystem Health Assessments in Korean Coastal Waters
}

\author{
Young-Ok Kim ${ }^{1}$ and Fu-Liu $\mathrm{Xu}^{2}$ \\ Guest editors of Special Issue
}

${ }^{1}$ South Sea Environment Research Division, South Sea Research Institute, KIOST, Geoje 656-834, Korea

${ }^{2}$ College of Urban and Environmental Sciences, Peking University, Beijing 100871, China

(c) KSO, KIOST and Springer 2014

Coastal pollution has long been recognized as a serious problem. Unfortunately, no simple solutions exist for sustaining a healthy ecosystem in coastal zones. Until now, approaches have only focused on affected areas and associated pollutants without much attention being paid to more preventative measures. A more comprehensive framework is appropriate for sustaining coastal marine health. Based on adequate policy making, a foundation for research could be established, and coastal environmental conditions could then be evaluated through regular assessments under new guidelines. The assessment results would provide instructive data for future decisions by policy makers concerning the restoration and maintenance of coastal health.

For the purpose of developing technical tools for assessing coastal ecosystem health conditions and providing practical ecological information, a project is currently being undertaken to develop the Marine Ecosystem Health Index (MEHI) based on organizing, simplifying, and generalizing the holistic data of marine ecosystems. During the last three years, 20102012, coastal ecosystem components were monitored in Jinhae Bay and Gwangyang Bay which are known to be highly polluted and which are special management areas in the South Sea of Korea. As part of the ongoing work to increase technical development, assessment indicators dealing with the relationships between chemical pressures and biological responses were selected and a monitoring system for indicator measurements was also established. Indicator criteria for determining healthy or unhealthy conditions in the ecosystem monitored were established based on the results analyzed

E-mail: yokim@kiost.ac,xufl@urban.pku.edu.cn from past data as well as the conditions monitored in this project. Finally, a numerical expression tool applying the selected indicators and the criteria was generated for the integration of bay ecosystem health assessment.

Water environments were studied in terms of eutrophication and sediment environments with regard to toxic organic compounds, and heavy metals were also monitored to understand the environmental conditions produced by chemical pollutants. Community structures and population dynamics of plankton and benthos were investigated at the same time, and these were intensely analyzed to understand the relationships with chemical factors. Each of these four subdivisions of water quality, sediment quality, plankton, and benthos was repectively expressed and integrated into a numerical expression to produce a health index or score. Finally, we were able to recognize which areas of Jinhae Bay and Gwangyang Bay are relatively healthy based on this assessment result and were able to prioritize actions to restore the health of the bay ecosystems. In a forthcoming step, the four indices will be integrated into a holistic health index of marine ecosystem assessments. The assessment results will be provided through an online service in the near future and will be shared with all concerned.

The project is a pioneering effort to assess the health of marine ecosystems in Korea and is continuously making efforts to ensure technical superiority with regard to application in other special management areas and to provide technical knowhow with regard to marine ecosystem health assessment based on the work carried out in Jinhae Bay and Gwangyang Bay (Picture 1). 


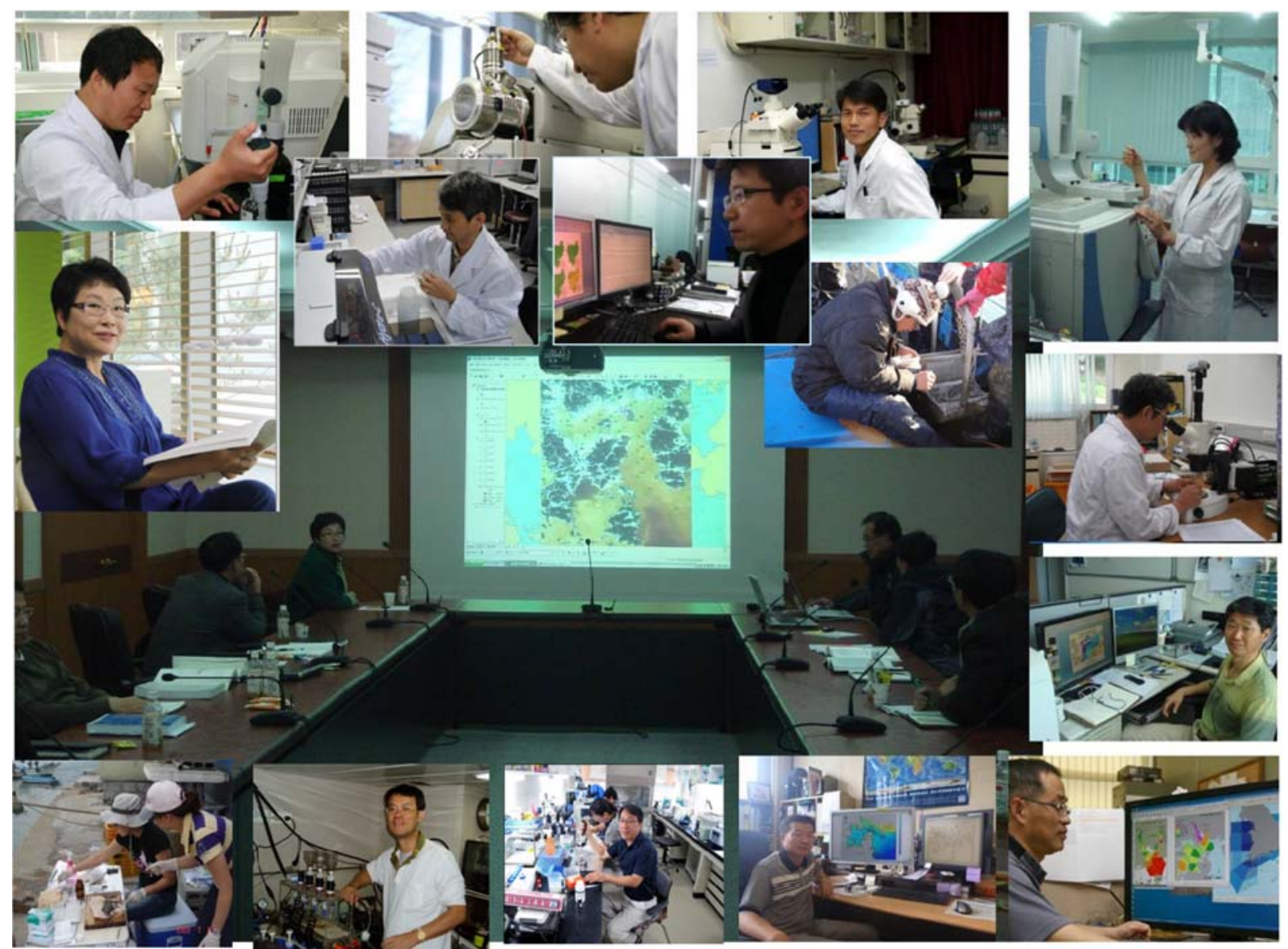

Picture 1. Major researchers for the project of marine ecosystem health assessment in Korea. Dr. Young-Ok Kim of project manager; Drs. Won-Joon Shim \& Sang Hee Hong for organic contaminants and Drs. Kyung-Tae Kim and Dong-Il Lim for heavy metals of the sediment quality; Drs. Dongseon Kim and Dong-Il Lim for water quality; Drs. Seungho Baek and Young-Ok Kim for plankton; Dr. Jin-Woo Choi and Dongsung Kim for benthos; Dr. Hyun-Woo Choi of GIS; Dr. Bhon Kyoung Koo for watershed; Dr. Jae Kwi So for water current; Dr. Dong-Lim Choi for geology; Dr. Jee Hyun Jeung for fish biomarker; Dr. Kwang-Sik Choi for shellfish pathogen

Lastly, we would like to thank OSJ for generously accepting the issue of this special edition as well as sponsors of the Korea Institute of Ocean Science Technology. Special thanks to the reviewers for their valuable revision suggestions on the paper. We sincerely hope that this special edition will encourage further scientific researches and the proper application of new assessment tools. 